\title{
What is Bioinformatics
}

Rajdeep Singh ${ }^{1}$ Somenath Dutta ${ }^{2}$

1Department of Biotechnology, DAV College, Sri Ganganagar, Rajasthan, India

${ }^{2}$ Center for Bioinformatics, Pondicherry Central University, Puducherry, India

\section{Abstract}

Bioinformatics is a new branch of the science world. Bioinformatics is a multidisciplinary approach. We use bioinformatics to understand biology information and save it into the biological database.

Apply data science on biological databases, discover a new drug, and modify extinction drugs to improve human life.

\section{Keywords}

Biology, Bioinformatics, Computer science, Software and tools, Coding.

\section{Introduction}

Bioinformatics is a multidisciplinary approach. Bioinformatics work jointly with different science brach to improve understanding and concept of biology.

Bioinformatics has recently been added to the science world. Bioinformatics comes with many opportunities for research to submit new research and build new products to improve human welfare.

The term Bioinformatics was invented by Paulien Hogeweg and Ben Hesper in 1970.

Margaret Oakley Dayhoff is the mother and father of Bioinformatics.

Bioinformatics name joins two-part of the word.

1. Bio means biology.

2. Informatique (a french word) means data processing.

In the Local internet language, if you like biology and coding. that is bio-informatics. 


\section{Definitions}

In Bioinformatics, many companies or people give different definitions. In the article, we list out some important definitions.

1. Bioinformatics is when biology, computer science, and information technology merge into a single discipline for managing and analyzing biological data using advanced computing techniques.

2. The combination of biology and information technology.

3. The stored data is available in the form of sequences and structures of proteins and nucleic acid.

4. The science of collecting and analyzing complex biological data such as genetic codes.

5. It involves the computational tool and method used to manage, analyze and manipulate volumes and volumes of biological data.

\section{Multidisciplinary approach}

The multidisciplinary approach means bioinformatics work with lots filed like computer science, biology, mathematics, biotechnology, statics, biochemistry, etc. 


\section{History}

Over a century ago, bioinformatics history started with an Austrian monk named Gregor Mendel. He is known as the "Father of Genetics." He clearly defined statistics and data studies on plants. after Mendel, many new scientists came and tried to find discoveries in the human world.

In the article, We discuss some major discoveries in the world.

1. The term bioinformatics was first introduced by Paulien Hogeweg and Ben Hesper in 1970.

2. In 1972, Paul berg made the first recombinant DNA molecule using the ligase. That same year, Stanley Cohen, Annie Chang, and Herbert Boyer produced the first recombinant DNA organism.

3. Joseph Sambrook led a team that refined DNA electrophoresis using agarose gel in 1975.

4. By 1977, a method for sequencing DNA was discovered, and the first genetic engineering company, Genetech, was founded.

5. In 1978, David Botstein discovered Restriction fragment length polymorphisms ( RFLP).

6. In 1980, Kary Mullis invented polymerase chain reaction (PCR)

7. In 1982, the Genetics computer group (GCG) was created as part of the University of Wisconsin biotechnology centre.

8. In 1986, The SWISS-PORT Database was created by the Department of medical biochemistry of geneva and the European molecular biology laboratory ( EMBL).

9. In 1987, the use of yeast artificial chromosomes (YAC) Was described.

10. In 1987, the human genome initiative was started.

11. In 1990, the BLAST program was implemented.

12. In 1991, the first time the term bioinformatic appeared in the scientific literature.

13. In 1992, a genetics linkage map of the entire human genome was published.

14. In 1996, Affymetrix produced the first commercial DNA chips.

15. In 1996, the cloned sheep Dolly was born on July 5, 1996.

16. In 1998, the swiss institute of biotechnology was established as a non-profit foundation.

17. In 1999, the human chromosome was completely sequenced.

18. In 2000, international research was sequenced.

19. In 2000, the international research consortium published chromosome 21.

20. In 2001, the human genome ( $3000 \mathrm{Mbp}$ ) was published

21. In 2003 the human genome project was completed in April 2003

22. In 2005, the genome sequence of rice was complete 


\section{Scope of bioinformatics}

Bioinformatics is a multidisciplinary approach. Bioinformatics combines many scientific fields. For that reason, the bioinformatics scope is extensive. Bioinformatics cover all major fields.

- Molecular Medicine

- Drug Therapy

- Microbial genome applications

- Forensic analysis of microbes

- Bio-weapon creation

- Biotechnology

- Biological computation

- Bio-engineering

- Biology

- Artificial intelligence

- Data mining

- Image processing

- Computational biology

- Statistics

- Mathematics

- Molecular biology

- Genetics

\section{Where Bioinformatics help}

- Experiment molecular biology

- In genetics and genomics

- Generating biological data

- Analysis of gene and proteins expression

- Comparison genomic data

- Simulation \& Modeling of DNA, RNA \& Protein 


\section{Software and tools in bioinformatics}

In bioinformatics lots of tools in the market, some free (open-source) and paid. In this field, I watch very few software as paid rest of free and open-source.

- $\underline{\text { BLAST }}$

- $\underline{\text { FAST }}$

- BioJS

- Biopython

- Bali-Phy

- BioPHP

All tools or software

https://en.wikipedia.org/wiki/List_of_bioinformatics_software

Open-Source software

https://en.wikipedia.org/wiki/List_of open-source bioinformatics software

\section{Language of bioinformatics}

In Bioinformatics 2, language is most commonly use it. But according to the company Requirements. Your programing language also changes. this recommendation is my expression base

1. R Language

2. Python Language

3. Other Language

\section{$\mathbf{R}$ Language}

$\mathbf{R}$ is a programming language. $\mathbf{R}$ is also used for statistical computing and graphics in build support by the $\mathrm{R}$ Foundation. The $\mathrm{R}$ language is widely used among statisticians and data miners for developing statistical software.

\section{Python Language}

Python is a general-purpose, high-level language. Python is used everywhere in the world. You use python and build anything. Python is used for machine learning, ai, data science, web development, desktop app design. Currently, python also rapidly grows in statistical analysis. 


\section{Other Language}

In another language category, we use the bash, $\mathrm{C}$, and $\mathrm{c}++$ language most commonly. But most of the time, real-world use of the $\mathrm{R}$ language of bio-information may change company to company based on company requirements. But Now, Days, python also grows rapidly.

\section{Role of Coding in Bioinformatics}

Two types of bioinformaticians in this world. First, which person knows about coding or software building skill, and second is the only use of software to predict and maybe show some kind result based on data use of the software. A non-coding person does not build their tool or software. They use other people to build software or tool.

\section{Lab in Bioinformatics}

Common two types of lab consider in bioinformatics science.

1. Dry lab

2. Wet lab

\section{Dry Lab:}

In Dry lab, we use computers to perform perdition based on biological data. We don't use chemicals, instruments and do not extract any DNA from cells. We use a laptop or desktop and good qualitative internet to analyze the data and give a prediction model.

\section{Wet Lab}

We use chemicals, cells, and instruments to get information and store it in the database inside the wet lab. With the help of these data, bioinformatician builds and predict the model base on data. 


\section{Operating systems}

The operating system is a basic fundamental tool for computer or laptop contact with the hardware. The most common operating system is three types.

1. Window

2. Mac OS

3. Linux Kernel

Window and Mac Os use the daily base widely used operating system. Window and Mac Os also provide a good UI interface for humans. Another hand Linux Kernel does not provide any $\mathrm{UI}$ interface. But Linux provides a command-line tool, which is more than important for us.

Linux also supports many distributions. Linux distributions help to provide a good UI interface for us. The most Commonly Linux distribution is Ubuntu.

\section{Why Use of Linux?}

Linux is very small in size and also runs with a small size of Ram and Storage.

\section{Where use of Linux?}

Right Now Linux Every Where. You use Linux to create a project with low device storage and Ram. Linux is a free and open-source project.

1. Most people Linux use in Android Mobile. Android is also built with Linux.

2. Linux is used to deploy websites on the Cloud. Every Cloud provides support for all Linux distributions.

\section{Why do we not use Windows or macOS in Bioinformatics?}

It depends from person to person and company to company. Most companies and persons like Linux in daily life, and They build bioinformatics and software also. Another person, like macOS or Windows to builds bioinformatics products. Both of the three systems work the same and design, build bioinformatics softwares.

The downfall of Windows and macOS.

1. Large Size

2. More Ram And Storge to perform the task. 


\section{Application of Bioinformatics}

Bioinformatics workaround biology and computer science. That reason the bioinformatics application list is huge. But I tell you a few of that. which are very important in bioinformatics

- Sequence mapping of biomolecules ( DNA, RNA, and Proteins).

- Finding of sites that restriction enzymes can cut

- Prediction of functional gene products

- Design 3d Structure of the molecule

- Designing software

- Developing device drivers

- Embedded solution

- Biological data management

- Data and image analysis

- Drug discovery

- Genomics and proteomics

\section{Conclusion}

Bioinformatics is a new branch of the science world. Many opportunities and discoveries in this field are pending.

Suppose you think and are inserted in bioinformatics. That is the right time to start learning. Right now, lots of YouTubers and courses are available on the internet. You just set your mind start learning. Your future is very bright in bioinformatics. 


\section{References}

https://en.wikipedia.org/wiki/Bioinformatics\#History

https://www.biologydiscussion.com/biodiversity/bioinformatics/bioinformatics-historycoverage-components-and-applications/11294

https://academic.oup.com/bib/article/20/6/1981/5066445

https://www.slideshare.net/AsadAfridi5/introduction-to-bioinformatics-147580009

https://www.slideshare.net/SomduttSharma4/bioinformatics-75142518

https://www.slideshare.net/JTADrexel/bioinformatics-2512758

https://www.slideshare.net/vidhyakalaivani29/major-databases-in-bioinformatics-71778405 